\title{
A NOTE ON A QUESTION OF BASS
}

BY

\author{
SANKAR P. DUTTA ${ }^{1}$
}

\begin{abstract}
In this paper we study the following question raised by Bass: Is a local ring with a finitely generated module of finite injective dimension Cohen-Macaulay? We prove that the answer is in the affirmative when a certain local cohomology of the ring is either decomposible or cyclic. We also study the above question in some special cases and some of its implications.
\end{abstract}

0. Introduction. In [B], Bass raised the question of the validity of the following:

CONJECTURE. If a local ring $A$ possesses a module $M$ of finite injective dimension, then $A$ is Cohen-Macaulay.

In this note we are going to describe a partial solution to this problem.

First, remarkable work was done by Peskine and Szpiro [PS]. They proved the conjecture for almost all geometrically important local rings containing a field. Their main tool of attack was the following conjecture which is known as "The intersection conjecture".

Conjecture. Let $\mathrm{A}$ be a local ring and let $T$ be a module of finite projective dimension over $A$. If $P$ is a prime ideal of $A$ with $l(A / P \otimes T)<\infty$, then $\operatorname{dim} A / P \leqslant \operatorname{proj} \operatorname{dim} T$.

Within a few years Hochster proved his famous theorem on existence of big Cohen-Macaulay (henceforth $\mathrm{C}-\mathrm{M}$ ) modules on local rings containing a field $[\mathbf{H}]$. $\mathrm{He}$ also showed there that existence of big $\mathrm{C}-\mathrm{M}$ modules implies the intersection conjecture which implies the above conjecture and thus finished the proof of the conjecture for all local rings containing a field. Using big C-M modules, Fossum, Foxby, Griffth and Reiten [FFGR] proved that for any module $M, \mu^{i}(M)>0$ for depth $M \leqslant i \leqslant \operatorname{inj} \operatorname{dim} M$. Their method of proof also showed that for any local ring $A$ the existence of a module of finite injective dimension implies depth $A \geqslant \operatorname{dim} A$ -1 .

In $\$ 1$ our main tool of attack is the dualizing complex. We prove the following theorem in 1.5 .

THEOREM 1. Let $A$ be a local ring of dimension $n$ and depth $n-r$. Let $M$ be $a$ module of finite injective dimension. Then $A$ is $C-M$ if

(i) $H_{m}^{n-r}(A)$ is decomposible,

(ii) $H_{m}^{n-r}(A)^{v}$ is cyclic.

Received by the editors October 16, 1984.

1980 Mathematics Subject Classification. Primary 13H10.

${ }^{1}$ Supported in part by NSF grant MCS-8108814(A02).

(c)1985 American Mathematical Society $0002-9947 / 85 \$ 1.00+\$ .25$ per page 
Here $v=\operatorname{Hom}(, E) . E$ is the injective hull of the residue field of $A$. (We will use this notation for the remainder of the paper.)

As a corollary to the method of proof used in the above theorem, we prove two more cases where $A$ has to be C-M, namely

(i) $\operatorname{dim} M>n-r$,

(ii) $M$ is cyclic (1.6).

The cases $\operatorname{dim} M=n$ and $M$ is cyclic were proved by Peskine and Szpiro in a totally different way. We also deduce the answer to the following question:

Given a local ring $A$, a module $T$ of finite length and finite projective dimension $d$, when is $T^{v} \simeq \operatorname{Ext}^{d}(T, A)$ ?

We show that equality holds $\Leftrightarrow A$ is Gorenstein (1.6). We also conclude in 1.6 that the conjecture follows directly from showing

$$
\operatorname{Tor}_{1}^{A}\left(T, \operatorname{Ext}^{r}(A, R)\right)=0 \Rightarrow \operatorname{Tor}_{2}^{A}\left(T, \operatorname{Ext}^{r}(A, R)\right)=0
$$

without using that intersection conjecture at all; here $\operatorname{depth} A=\operatorname{dim} A-r$ and $\operatorname{Ext}^{r}(A, R)=H_{m}^{n-r}(A)^{v}$.

In $\S 2$ we use the techniques of intersection multiplicity of modules to prove the following theorem (see 2.3).

TheOREM 2. Let $A$ be a complete equidimensional local ring of dimension 4 and let $A$ possess a module of finite injective dimension. Then $A$ is $C-M$ if either

(i) depth $H_{m}^{3}(A)^{v}>0$, or

(ii) $l\left(H_{m}^{3}(A)\right)<\infty$ and $H_{m}^{3}(A) \simeq H_{M}^{3}(A)^{v}$.

As a corollary we deduce that a complete local U.F.D. with a module of finite injective dimension is Gorenstein if

(i) $H_{1}(\mathbf{x} ; A)$ (the Koszul homology) is cyclic, or

(ii) $\operatorname{dim} A=4$.

Throughout this paper all rings are commutative noetherian with identity and all modules are finitely generated (with the possible exception of injective modules and local cohomology modules). Because of the nature of our problem, we will also assume, without any loss of generality, that $A$ is complete and that the depth of the module of finite injective dimension is zero.

I wish to thank M. Hochster for suggesting that I study the case when $l(M)<\infty$ and proj $\operatorname{dim} M<\infty$.

1.

1.1 Through this section $\operatorname{dim} A=n$, $\operatorname{depth} A=n-r$, and $M$ is a finitely generated module of finite injective dimension of depth zero. (Since the author is interested in exploring the significant role a dualizing complex might play in such problems, that the depth $A$ can be taken to be $n-1$, a consequence of the existence of big $\mathrm{C}-\mathrm{M}$ modules, is not assumed in this section.)

We will frequently use the following fact:

$$
\operatorname{Tor}_{i}^{A}(N, I) \simeq \operatorname{Hom}\left(\operatorname{Ext}^{i}(N, A), I\right)
$$

for every injective module $I$. For a proof see [AB, p. 54]. 
Let $R$ be a complete Gorenstein ring with $A=R / I$ and $\operatorname{dim} R=\operatorname{dim} A$.

Peskine and Szpiro have shown [PS] that given $M$, a module of finite injective dimension, there exists a module $T$ of finite projective dimension with the following properties:

$$
\begin{aligned}
& \text { (i) } T=\operatorname{Ext}_{A}^{n-r}(E, M), \\
& \text { (ii) } \operatorname{Ext}_{A}^{i}(T, A)=\operatorname{Ext}_{R}^{r+i}(M, R), \\
& \text { (iii) } \operatorname{supp} T=\operatorname{supp} M .
\end{aligned}
$$

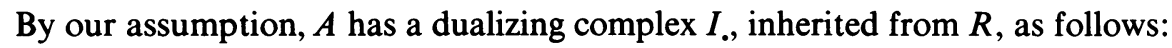

$$
\begin{aligned}
& I .: 0 \rightarrow I_{0} \stackrel{\phi_{0}}{\rightarrow} I_{1} \stackrel{\phi_{1}}{\rightarrow} \cdots \stackrel{\phi_{n-1}}{\rightarrow} I_{n} \rightarrow 0, \\
& I_{i}=\bigoplus_{\operatorname{dim} A / P=n-i} E(A / P), \quad H^{i}\left(I_{.}\right)=\operatorname{Ext}_{R}^{i}(A, R), \quad I_{n}=E ;
\end{aligned}
$$

we write $B_{i+1}=\operatorname{Im} \phi_{i}, Z_{i}=\operatorname{ker} \phi_{i}$. Then we get from (3) the exact sequences

$$
\begin{aligned}
& 0 \rightarrow A^{*} \rightarrow I_{0} \rightarrow B_{1} \rightarrow 0, \\
& 0 \rightarrow B_{1} \rightarrow Z_{1} \rightarrow \operatorname{Ext}_{R}^{1}(A, R) \rightarrow 0, \\
& 0 \rightarrow Z_{1} \rightarrow I_{1} \rightarrow B_{2} \rightarrow 0, \\
& \cdots \\
& 0 \rightarrow B_{r} \rightarrow Z_{r} \rightarrow \operatorname{Ext}_{R}^{r}(A, R) \rightarrow 0, \\
& 0 \rightarrow Z_{r} \rightarrow I_{r} \rightarrow I_{r+1} \rightarrow \cdots \rightarrow I_{n} \rightarrow 0 .
\end{aligned}
$$

From now on we will not put any subscript or superscript for Tor and Ext computed over $A$.

1.2 We prove the following two lemmas.

LEMMA 1. For any module $N, \operatorname{Ext}^{j}\left(I_{i}, N\right)=0$ for $i \neq n$.

Proof. $\operatorname{Ext}^{j}\left(I_{i}, N\right)=\operatorname{Tor}_{j}\left(I_{i}, N^{v}\right)^{v}$ since $N^{v}$ is an artinian module $N^{v}=\lim _{\rightarrow} N_{\alpha}$, with $L\left(N_{\alpha}\right)<\infty$. Hence

$$
\operatorname{Tor}_{j}\left(I_{i}, N^{v}\right)=\underset{\lim }{\rightarrow} \operatorname{Tor}_{j}\left(I_{i}, N_{\alpha}\right)=\underset{\lim }{\rightarrow} \operatorname{Hom}\left(\operatorname{Ext}^{j}\left(N_{\alpha}, A\right), I_{i}\right)=0
$$

for $i \neq n$ since $l\left(\operatorname{Ext}^{j}\left(N_{\alpha}, A\right)\right)<\infty$.

LEMMA 2. $\operatorname{Tor}_{j}\left(T, I_{i}\right)=0$ for $r+j>i$.

Proof. We have

$$
\begin{aligned}
\operatorname{Tor}_{j}\left(T, I_{i}\right) & =\operatorname{Hom}\left(\operatorname{Ext}^{j}(T, A), I_{i}\right) \\
& =\operatorname{Hom}\left(\operatorname{Ext}_{R}^{r+j}(M, R), I_{i}\right) \text { by (2) } \\
& =0
\end{aligned}
$$

since $R$ is Gorenstein, $\operatorname{dim} \operatorname{Ext}_{R}^{r+j}(M, R) \leqslant n-r-j$.

1.3 Proposition. With the notations as above we have the following relations:

(i) $T=\operatorname{Ext}^{n-r}(E, M)=\operatorname{Hom}\left(\operatorname{Ext}_{R}^{r}(A, R), M\right)$,

(ii) $\operatorname{Tor}_{1}\left(T, \operatorname{Ext}_{R}^{r}(A, R)\right)=0$ and $\operatorname{Tor}_{2}\left(T, \operatorname{Ext}_{R}^{r}(A, R)\right)=T \otimes \operatorname{Ext}_{R}^{r-1}(A, R)$,

(iii) $\operatorname{Ext}^{1}\left(\operatorname{Ext}_{R}^{r}(A, R), M\right)=0$,

(iv) $T \otimes \operatorname{Ext}_{R}^{r}(A, R)=M$. 
Proof. (i) and (iii) From $0 \rightarrow Z_{r} \rightarrow I_{r} \rightarrow \cdots \rightarrow I_{n}=E \rightarrow 0$ in (4), we get $\operatorname{Ext}^{n-r}(E, M)=\operatorname{Hom}\left(Z_{r}, M\right)$ by Lemma 1 of 1.2 .

From $0 \rightarrow B_{r} \rightarrow Z_{r} \rightarrow \operatorname{Ext}_{R}^{r}(A, R) \rightarrow 0$, we get

$$
\begin{aligned}
0 & \rightarrow \operatorname{Hom}\left(\operatorname{Ext}_{R}^{r}(A, R), M\right) \rightarrow \operatorname{Hom}\left(Z_{r}, M\right) \rightarrow \operatorname{Hom}\left(B_{r}, M\right) \\
& \rightarrow \operatorname{Ext}^{1}\left(\operatorname{Ext}_{R}^{r}(A, R), M\right) \rightarrow \operatorname{Ext}^{1}\left(Z_{r}, M\right) \rightarrow \cdots .
\end{aligned}
$$

Since $\operatorname{Hom}\left(B_{r}, M\right) \hookrightarrow \operatorname{Hom}\left(I_{r-1}, M\right)$ by Lemma 1 of $1.2, \operatorname{Hom}\left(B_{r}, M\right)=0$.

Again $\operatorname{Ext}^{1}\left(Z_{r}, M\right) \simeq \operatorname{Ext}_{A}^{n-r+1}(E, M)$ by Lemma 1 of 1.2. Since inj $\operatorname{dim} M=$ depth $A=n-r$, we get $\operatorname{Ext}^{1}\left(Z_{r}, M\right)=0$. Thus from the above long exact sequence, we get (i) and (iii).

(ii) Applying $\otimes T$ to $0 \rightarrow B_{r} \rightarrow Z_{r} \rightarrow \operatorname{Ext}_{R}^{r}(A, R) \rightarrow 0$, we get

$$
\cdots \rightarrow \operatorname{Tor}_{1}\left(T, Z_{r}\right) \rightarrow \operatorname{Tor}_{1}\left(T, \operatorname{Ext}_{R}^{r}(A, R)\right) \rightarrow T \otimes B_{r} \rightarrow \cdots .
$$

Since $T \otimes I_{r-1} \rightarrow T \otimes B_{r} \rightarrow 0$ is exact and $T \otimes I_{r-1}=0$ by Lemma 2 of 1.2 , we have $T \otimes B_{r}=0$. Now by Lemma 2 of 1.2 and from $0 \rightarrow Z_{r} \rightarrow I_{r} \rightarrow \cdots \rightarrow I_{n}=E$ $\rightarrow 0$, we get $\operatorname{Tor}_{1}\left(T, Z_{r}\right)=\operatorname{Tor}_{n-r+1}(T, E)=0$ [proj $\left.\operatorname{dim} T=n-r\right]$.

Thus $\operatorname{Tor}_{1}\left(T, \operatorname{Ext}_{R}^{1}(A, R)\right)=0$. We have in (4)

$$
\begin{aligned}
& 0 \rightarrow B_{r-1} \rightarrow Z_{r-1} \rightarrow \operatorname{Ext}_{R}^{r-1}(A, R) \rightarrow 0, \\
& 0 \rightarrow Z_{r-1} \rightarrow I_{r-1} \rightarrow B_{r} \rightarrow 0, \\
& 0 \rightarrow B_{r} \rightarrow Z_{r} \rightarrow \operatorname{Ext}^{r}(A, R) \rightarrow 0 .
\end{aligned}
$$

By applying $\otimes T$ to these short exact sequences, it follows that

$$
\operatorname{Tor}_{2}\left(T, \operatorname{Ext}_{R}^{r}(A, R)\right) \simeq \operatorname{Tor}_{1}\left(T, B_{r}\right) \simeq T \otimes Z_{r-1} \simeq T \otimes \operatorname{Ext}_{R}^{r-1}(A, R)
$$

(Lemma 2 of 1.2). M.

(iv) Let $J^{\bullet}: 0 \rightarrow J^{0} \rightarrow J^{1} \rightarrow \cdots \rightarrow J^{n-r} \rightarrow 0$ be a minimal injective resolution of Applying $H_{m}^{0}$ to the above sequence, we get

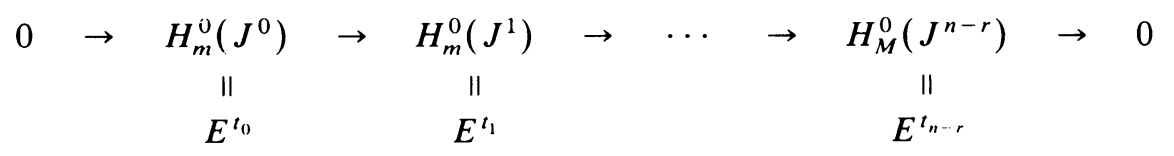

where $t_{i}=\mu^{i}(M)$, such that the $i$ th homology of this sequence is $H_{m}^{i}(M)$. Applying $\operatorname{Hom}(E,-)$ to the above sequence, we get

$$
F^{\bullet}: 0 \rightarrow A^{t_{0}} \rightarrow A^{t_{1}} \rightarrow \cdots \rightarrow A^{t_{n-r}} \rightarrow 0,
$$

a minimal projective resolution of $T$ [PS].

Let $\left(F^{*}\right)^{*}$ denote the complex $\operatorname{Hom}\left(F^{*}, A\right)$. It is obvious that $H_{m}^{0}\left(I_{0}\right)^{v}=\left(F^{*}\right)^{*}$. In order to make our dualizing complex $I_{.}: 0 \rightarrow I_{0} \rightarrow \cdots \rightarrow I_{n} \rightarrow 0$ normalized, we change the suffixes and write it as

$$
I^{\bullet}: 0 \rightarrow I^{-n} \rightarrow I^{-(n-1)} \rightarrow \cdots \rightarrow I^{0} \rightarrow 0, \quad I^{-k}=\bigoplus_{\operatorname{dim}} \bigoplus_{P=k} E(A / P) .
$$

Since $F^{\cdot}$ is a complex with $F^{i}=0$ for $i<0$, we can write $\left(F^{*}\right)^{*}$ as a complex with $F^{-i}=A^{t_{i}}$ and $F^{i}=0$ for $i>0$. We know that $\operatorname{Hom}\left(\left(F^{*}\right)^{*}, I^{*}\right)$ is quasi-isomorphic to $M[\mathbf{H a}]$. 
We consider the double complex $\operatorname{Hom}\left(\left(F^{*}\right)^{*}, I^{\bullet}\right)$ :

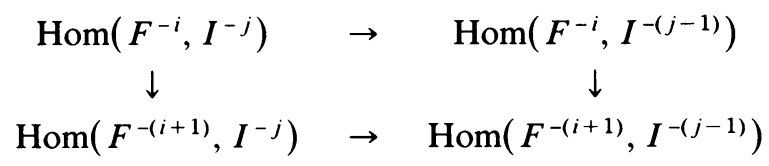

We know the 0th homology of this double complex is $M$.

Starting with the homologies of the horizontal rows first, we see that

$$
E_{2}^{-i,-j}=\operatorname{Tor}_{n-r-i}\left(T, \operatorname{Ext}_{R}^{n-j}(A, R)\right) ;
$$

hence

$$
E_{2}^{-i,-i}=\operatorname{Tor}_{n-r-i}\left(T, \operatorname{Ext}_{R}^{n-i}(A, R)\right)
$$

for $i>n-r$,

$$
E_{2}^{-i,-i}=0 \quad(\because \operatorname{proj} \operatorname{dim} T=n-r)
$$

for $i<n-r$,

$$
E_{2}^{-i,-i}=0 \quad\left(\because \operatorname{Ext}^{i}(A, R)=0, i>r\right)
$$

for $i=n-r$,

$$
\operatorname{Ext}_{2}^{-(n-r),-(n-r)}=T \otimes \operatorname{Ext}^{r}(A, R) .
$$

Since the above spectral sequence converges to the homology of the double complex above, it can be easily checked that $T \otimes \operatorname{Ext}_{R}^{r}(A, R) \simeq M$.

1.4 Remarks. (1) Property (ii) of the above proposition can also be derived from the spectral sequence described above.

(2) It can easily be shown that the map

$$
T \otimes \operatorname{Ext}_{R}^{r}(A, R) \rightarrow M, \quad f \otimes \alpha \rightarrow f(\alpha)
$$

is actually an isomorphism.

(3) Properties (i) and (iv) of the above proposition show that one can easily construct a module with the same properties as the one is Sharp's construction [S] starting from Peskine-Szpiro's construction, in a very elegant way. Moreover from Peskine-Szpiro's construction, one derives (ii) and (iii) of the above proposition, which are extremely useful. It also possesses property (ii) of (2) which has already been used many times to calculate Tor and Ext.

At first I started working on the case of a module of finite length and finite projective dimension. By applying the spectral sequence obtained from change of base from $R$ to $A$, I arrived at Proposition 1.3. Then as I tried to generalize the results for any module of finite injective dimension, I came across Sharp's construction-but since his approach was rather far from my approach, I started working with Peskine-Szpiro's construction and Hartshorne's result ( $\$ 6$, Chapter V of [Ha]) really helped to get all that I needed in the process of generalization.

1.5 Proof of Theorem 1. (i) In view of the above proposition, the proof of (i) becomes rather trivial. We know that $H_{m}^{i}(A)^{v}=\operatorname{Ext}_{R}^{n-i}(A, R)$. Thus $H_{m}^{n-r}(A)^{v}=$ $\operatorname{Ext}_{R}^{r}(A, P)$. Since $A$ is complete we can assume, without loss of generality, that $M$ is indecomposable. Because of Proposition 1.3 (iv), $\operatorname{Ext}_{R}^{r}(A, R)$ must be indecomposable. (This implies $H_{m}^{n-r}(A)$ must be indecomposable.) 
(ii) Properties (i) and (iv) of Proposition 1.3 imply that both ann ${ }_{A} M$ and ann ${ }_{A} T$ contain $\operatorname{ann}_{A} \operatorname{Ext}^{r}(A, R)$. Therefore when $\operatorname{Ext}_{R}^{r}(A, R)$ is cyclic, $T \simeq M$. Let $\operatorname{Ext}_{R}^{r}(A, R)=A / I$. We consider the exact sequence

$$
0 \rightarrow I \rightarrow A \rightarrow A / I \rightarrow 0 \text {. }
$$

Since $\operatorname{Tor}_{1}\left(T, \operatorname{Ext}^{r}(A, R)=0\right)$, applying $T$ to the above sequence, we get

$$
0 \rightarrow T \otimes I \rightarrow T \rightarrow T \rightarrow 0 .
$$

This implies that $T \otimes I=0$, which is a contradiction since $A$ is local.

1.6 Corollaries. (1) The above arguments also show that since $\operatorname{dim} M \leqslant$ $\operatorname{dim} \operatorname{Ext}_{R}^{r}(A, R)$, if $\operatorname{dim} M>n-r$, then $A$ is $C-M$. (If $A$ is a domain, if $\operatorname{dim} M>n$ $-r-1$, then $A$ is $C-M$.)

(2) When $M$ is cyclic, the relation $T \otimes \operatorname{Ext}_{R}^{r}(A, R)=M$ implies $\operatorname{Ext}^{r}(A, R)$ is cyclic. Hence, by the theorem above, $A$ is $C-M$.

That $A$ is actually Gorenstein follows from the next corollary.

(3) Let $T$ be a module of finite length and finite projective dimension over a local ring A. Let $d=$ proj $\operatorname{dim} T$. M. Hochster asked me the following:

Question: When is $M^{v} \simeq \operatorname{Ext}^{d}(M, A)$ ?

Answer: The isomorphism holds good if and only if $A$ is Gorenstein.

Proof. $A$ Gorenstein implies $M^{v} \simeq u \operatorname{Ext}^{d}(M, A)$ since in this case $d=\operatorname{dim} A=$ depth $A$, and $A$ is a canonical module over itself.

$\Leftarrow$ : Suppose $M^{v} \simeq \operatorname{Ext}^{d}(M, A)$.

Actually from the construction of $T$ from $M^{v}$ (since $M^{v}$ has finite injective dimension) it follows that $T=\operatorname{Ext}^{d}(M, A)$. Since we know $M^{v} \simeq \operatorname{Ext}^{d}(M, A) \otimes$ $\operatorname{Ext}_{R}^{r}(A, R)$ (Proposition 1.3), $\operatorname{Ext}_{R}^{r}(A, R)$ is cyclic and hence by the theorem above $A$ is C-M. Let $\Omega$ be a canonical module of $A$. Then it can be shown that $M^{\prime \prime} \simeq \operatorname{Ext}^{d}(M, A) \otimes \Omega$. But this implies $\Omega$ is cyclic and hence isomorphic to $A$. Thus $A$ is Gorenstein.

REMARK. From (4) we get a family of relations only a couple of which have been mentioned in (ii) of Proposition 1.3. In view of one of the consequences of the counterexample to the generalized intersection multiplicity conjecture, the rigidity conjecture is now under a shadow of doubt. But we do not need the whole rigidity conjecture to prove Bass's conjecture. We have already shown $\operatorname{Tor}_{1}\left(T, \operatorname{Ext}^{r}(A, R)\right)$ $=0$. If we could somehow show that this (or otherwise) implies $\operatorname{Tor}_{2}^{A}\left(T, \operatorname{Ext}^{r}(A, R)\right.$ ) $=0$, then from the relations mentioned above we can, very easily, arrive at a contradiction. And that is what happens when $\operatorname{dim} A=3$ (one does not have to use the intersection conjecture at all). But in spite of my best efforts, I am unable to prove this at the present moment, even assuming depth $A=n-1$.

2. In this section we assume depth $A=n-1$. Then the dualizing complex can be broken down into exact sequences as follows:

$$
\begin{aligned}
& 0 \rightarrow A^{*} \rightarrow I^{0} \rightarrow B_{1} \rightarrow 0, \\
& 0 \rightarrow B_{1} \rightarrow Z_{1} \rightarrow \operatorname{Ext}^{1}(A, R) \rightarrow 0, \\
& 0 \rightarrow Z_{1} \rightarrow I_{1} \rightarrow I_{2} \rightarrow \cdots \rightarrow I_{n} \rightarrow 0 .
\end{aligned}
$$


The relations (ii) and (iii) of Proposition 1.3 become more explicit. We actually get

(ii) ${ }^{\prime} \operatorname{Tor}_{i}\left(T, \operatorname{Ext}^{1}(A, R)\right) \simeq \operatorname{Tor}_{i-2}(T, A), i \geqslant .1$,

(iii) $\operatorname{Ext}^{i}\left(\operatorname{Ext}_{R}^{1}(A, R), M\right) \simeq \operatorname{Ext}_{A}^{i-2}\left(A^{*}, M\right), i \geqslant 1$.

Here $A^{*}=\operatorname{Hom}(A, R)$. Let $M$ be a module of finite length and finite injective dimension. Then $l(T)<\infty$, proj $\operatorname{dim} T=n-1$.

2.1 Proposition. Let $A, T$ be as above with $A$ equidimensional. Let $Q$ be a module with $\operatorname{dim} Q \leqslant 1$. Then $\chi^{A}(T, Q)$ is 0

$$
\left(\chi^{A}(T, Q)=\sum_{i=0}^{n-1}(-1)^{i} l\left(\operatorname{Tor}_{i}(T, Q)\right)\right) .
$$

Proof. If $l(Q)<\infty, \chi^{A}(T, Q)=l(Q) \chi^{A}(T, A / m)=0$; here $m$ is the maximal ideal of $A$. Hence we can assume $Q=A / P, \operatorname{dim} A / P=1, P$ a prime ideal of $A$. Let $\left\{x_{1}, x_{2}\right\}$ be an $A$-sequence contained in $\operatorname{ann}_{A} T$ such that one of them is $A / P$-regular.

Now, $\chi\left(T, A / P^{n}\right)=l\left(A_{P} / P^{n} A_{P}\right) \chi(T, A / P)$ (since in any prime filtration of $A / P^{n}, A / P$ occurs $l\left(A_{P} / P^{n} A_{P}\right)$ times and the remaining quotients are isomorphic to $A / m$ ).

Hence

$$
\chi(T, A / P)=\lim _{n \rightarrow \infty} \chi\left(T, A / P^{n}\right) / l\left(A_{P} / P^{n} A_{P}\right) .
$$

From the exact sequence

$$
0 \rightarrow P^{(n)} / P^{n} \rightarrow A / P^{n} \rightarrow A / P^{(n)} \rightarrow 0
$$

we get

$$
\chi\left(T, A / P^{n}\right)=\mathrm{X}\left(T, A / P^{(n)}\right) \quad\left(\text { since } l\left(P^{(n)} / P^{n}\right)<\infty\right)
$$

Hence

$$
\chi(T, A / P)=\lim _{n \rightarrow \infty} \chi\left(T, A / P^{(n)}\right) / l\left(A_{P} / P^{n} A_{P}\right) .
$$

We write $\bar{A}=A /\left(x_{1}, x_{2}\right)$ an consider the exact sequence

$$
0 \rightarrow N \rightarrow \bar{A}^{t_{n-4}} \rightarrow \cdots \rightarrow \bar{A}^{t_{1}} \rightarrow \bar{A}^{t_{0}} \rightarrow T \rightarrow 0 .
$$

Here $\operatorname{dim} N=n-2$ and depth $N=n-3$. Hence

$$
\chi(T, A / P)= \pm \chi(N, A / P)= \pm \chi\left(N, A / P^{(n)}\right) / l\left(A_{P} / P^{n} A_{P}\right) .
$$

Now

$$
\chi\left(N, A / P^{(n)}\right)=l\left(N \otimes A / P^{(n)}\right)-l\left(\operatorname{Tor}_{1}^{A}\left(N, A / P^{(n)}\right)\right) .
$$

(Since proj $\operatorname{dim} N=2$ and $\operatorname{ann}_{A} N$ contains an $A / P^{(n)}$-regular element, $\operatorname{Tor}_{2}^{A}\left(N, A / P^{(n)}\right)=0$.) Because of the exact sequence $A / P^{n} \rightarrow A / P^{(n)} \rightarrow 0$ and $\left(A /\left(x_{1}, x_{2}\right)\right)^{t} \rightarrow N \rightarrow 0$, we get

$$
l\left(N \otimes A / P^{(n)}\right) \leqslant t l\left(A /\left(x_{1}, x_{2}\right) \otimes A / P^{n}\right) \leqslant K \cdot t^{n-2},
$$

where $K$ is a suitable constant (since $\left.\operatorname{dim} A /\left(x_{1}, x_{2}\right)=n-2\right)$.

As $l\left(A_{P} / P^{n} A_{P}\right)$ is a polynomial of degree $n-1$ for $n \gg 0$, we have from above

$$
\lim _{n \rightarrow \infty} l\left(N \otimes A / P^{(n)}\right) / l\left(A_{P} / P^{n} A_{P}\right)=0 .
$$


Again from the exact sequence $0 \rightarrow G \rightarrow\left(A /\left(x_{1}, x_{2}\right)\right)^{t} \rightarrow N \rightarrow 0$ we get, by applying $\otimes A / P^{(n)}$,

$$
\begin{aligned}
l\left(\operatorname{Tor}_{1}\left(N, A / P^{(n)}\right)\right) & \leqslant l\left(G \otimes A / P^{(n)}\right)+t l\left(\operatorname{Tor}_{1}\left(A /\left(x_{1}, x_{2}\right), A / P^{(n)}\right)\right) \\
& =l\left(G \otimes A / P^{(n)}\right)+t l\left(A /\left(x_{1}, x_{2}\right) \otimes A / P^{(n)}\right) .
\end{aligned}
$$

By reasoning as above it follows that

$$
\lim _{n \rightarrow \infty} l\left(\operatorname{Tor}_{1}^{A}\left(N, A / P^{(n)}\right)\right) / l\left(A_{P} / P^{n} A_{P}\right)=0 .
$$

From equations (5)-(9) the required result follows.

2.2 Proposition. Let $A, T$ be as above and let $\operatorname{dim} A=4$. Let $Q$ be a module of dimension 2 and let $T \simeq \operatorname{Ext}^{3}(T, A)$. Then $\chi(T, Q)=0$.

Proof. Since $\chi(T, N)=0$ if $\operatorname{dim} N=1$, it is enough to show $\chi(T, A / P)=0$ with $\operatorname{dim} A / P=2$. Let $H$ be the integral closure of $A / P$. Then $H$ is C-M of dimension 2. Moreover, $\chi(T, A / P)=0$ if and only if $\chi(T, H)=0$ (by the previous proposition). Let $\bar{H}$ denote $\operatorname{Ext}_{R}^{2}(H, R)$. Now

$$
\begin{aligned}
\chi(T, H) & =l(T \otimes H)-l\left(\operatorname{Tor}_{1}^{A}(T, H)\right), \\
l(T \otimes H) & =l\left(\operatorname{Ext}_{R}^{4}(T \otimes H, R)\right)=l\left(\operatorname{Ext}^{2}(T, \bar{H})\right)=l\left(\operatorname{Tor}_{1}(T, \bar{H})\right) .
\end{aligned}
$$

Hence $\chi(T, H)=-\chi(T, \bar{H})$. But $H$ and $\bar{H}$ have the same minimal primes occurring an equal number of times in any prime filtration. Hence, by the previous proposition, $\chi(T, H)=\chi(T, \bar{H})$. Thus $\chi(T, H)=0$.

2.3 Proof of Theorem 2. Since $A$ is complete and equidimensional by induction on $\operatorname{dim} M$ we reduce to proving the theorem when $l(M)<\infty$. Hence $l(T)<\infty$. Without any loss of generality, we can assume $T \simeq \operatorname{Ext}^{3}(T, A)$. We know that $H_{m}^{3}(A)^{v} \simeq \operatorname{Ext}_{R}^{1}(A, R)$.

(i) depth of $\operatorname{Ext}_{R}^{1}(A, R)>0$. Since proj $\operatorname{dim} T=3$ and depth of $\operatorname{Ext}_{R}^{1}(A, R)=1$, we have $\operatorname{Tor}_{3}\left(T, \operatorname{Ext}_{R}^{1}(A, R)\right)=0$. By Proposition 1.3 we also have $\operatorname{Tor}_{1}\left(T, \operatorname{Ext}_{R}^{1}(A, R)\right)=0$. Hence

$$
\chi\left(T, \operatorname{Ext}_{R}^{1}(A, R)\right)=l\left(T \otimes \operatorname{Ext}_{R}^{1}(A, R)\right)+l\left(\operatorname{Tor}_{2}^{A}\left(T, \operatorname{Ext}_{R}^{1}(A, R)\right)\right) \neq 0 .
$$

On the other hand, this is a contradiction since, by the above proposition, $\chi\left(T, \operatorname{Ext}_{R}^{1}(A, R)\right)=0$. (The case depth $\operatorname{Ext}_{R}^{1}(A, R)=2$ is left as an easy exercise.)

(ii) $\operatorname{dim} \operatorname{Ext}_{R}^{1}(A, R)=0$ and $\operatorname{Ext}_{R}^{1}(A, R)=\operatorname{Ext}_{R}^{1}(A, R)^{v}$. As above, in this case

$$
\begin{aligned}
\chi\left(T, \operatorname{Ext}_{R}^{1}(A, R)\right)= & l\left(T \otimes \operatorname{Ext}_{A}^{1}(A, R)\right)+l\left(\operatorname{Tor}_{2}^{A}\left(T, \operatorname{Ext}_{R}^{1}(A, R)\right)\right) \\
& -l\left(\operatorname{Tor}_{3}^{A}\left(T, \operatorname{Ext}_{R}^{1}(A, R)\right)\right) .
\end{aligned}
$$

Now

$$
\begin{aligned}
l\left(\operatorname{Tor}_{3}^{A}\left(T, \operatorname{Ext}_{R}^{1}(A, R)\right)\right) & =l\left(\operatorname{Hom}\left(\operatorname{Ext}^{3}(T, A), \operatorname{Ext}_{R}^{1}(A, R)\right)\right) \\
& =l\left(T \otimes \operatorname{Ext}_{R}^{4}\left(\operatorname{Ext}_{R}^{1}(A, R), R\right), E\right) \\
& =l\left(T \otimes \operatorname{Ext}_{R}^{1}(A, R), E\right)
\end{aligned}
$$

by assumption. Hence from (10)

$$
\left.\chi\left(T, \operatorname{Ext}_{R}^{1}(A, R)\right)=l\left(\operatorname{Tor}_{2}^{A}\left(T, \operatorname{Ext}_{R}^{1}(A, R)\right)\right)=l\left(T \otimes A^{*}\right) \quad \text { by (ii) }\right)^{\prime} .
$$


But, by Proposition 2.1, $\chi\left(T, \operatorname{Ext}_{R}^{1}(A, R)\right)=0$. Hence $T \otimes A^{*}$ must be zero, which is a contradiction.

2.4 Corollaries. Here we assume A to be complete or a quotient of a Gorenstein ring.

(1) Let $A$ be U.F.D. with a module $M$ of finite injective dimension. Then $A$ is $C-M$ in the following cases:

(i) $H_{1}(\mathbf{x} ; A)$ for some (every) system of parameters $\mathbf{x}$ is cyclic.

(ii) $\operatorname{dim} A=4, A$ is complete.

Proof of (i). We can take, without any loss of generality, $\mathbf{x}$ such that the first $n-1$ elements $x_{1}, \ldots, x_{n-1}$ form an $A$-sequence. It can easily be seen that

$$
\begin{aligned}
H_{1}(\mathbf{x} ; A)^{v} & \simeq \operatorname{Ext}_{R}^{n}\left(A /\left(x_{1}, \ldots, x_{n-1}\right), R\right) \\
& \simeq \operatorname{Ext}^{1}(A, R) /\left(x_{1}, \ldots, x_{n-1}\right) \operatorname{Ext}^{1}(A, R),
\end{aligned}
$$

where $R$ is as described in $\S 1$. Since $A$ is U.F.D., $\operatorname{Hom}(A, R) \simeq A^{*} \simeq A$. Hence applying $\operatorname{Hom}(-, R)$ to $0 \rightarrow I \rightarrow R \rightarrow A \rightarrow 0$, we get $0 \rightarrow A \rightarrow R \rightarrow I^{*} \rightarrow$ $\operatorname{Ext}_{R}^{1}(A, R) \rightarrow 0$. Since $\operatorname{depth} A=n-1$, depth $I=n=\operatorname{depth} I^{*}$. Thus $\operatorname{Ext}^{3}\left(\operatorname{Ext}_{R}^{1}(A, R), R\right) \simeq \operatorname{Ext}_{R}^{1}(A, R)$, i.e., $\operatorname{Ext}_{R}^{1}(A, R)$ is a C-M module of dimension $n-3$ which is, moreover, self-dual. We choose $\mathbf{x}$ in such a way that $\left\{x_{1}, \ldots, x_{n-3}\right\}$ is an $\operatorname{Ext}^{1}(A, R)$-sequence as well and

$$
\left\{x_{n-2}, x_{n-1}, x_{n}\right\} \subset \operatorname{ann} \operatorname{Ext}_{R}^{1}(A, R) /\left(x_{1}, \ldots, x_{n-3}\right) \operatorname{Ext}_{R}^{1}(A, R) .
$$

Therefore from (11) we conclude that

$$
H_{1}(\mathbf{x} ; A)=\operatorname{Ext}_{R}^{1}(A, R) / \mathbf{x} \operatorname{Ext}_{R}^{1}(A, R)
$$

and hence $\operatorname{Ext}_{R}^{1}(A, R)$ is cyclic. Now by Theorem 1 we are done.

Proof of (ii). When $\operatorname{dim} A=4, \operatorname{dim} \operatorname{Ext}_{R}^{1}(A, R)=1$, and it is C-M as mentioned above. Hence $\operatorname{Tor}_{3}^{A}\left(T, \operatorname{Ext}_{R}^{1}(A, R)\right)=0$ for any module $T$ of finite length and finite projective dimension. Let $l(M)<\infty$ and let $T$ be as described in $\S 1$. Thus

$$
\chi\left(T, \operatorname{Ext}_{R}^{1}(A, R)\right)=l\left(T \otimes \operatorname{Ext}_{R}^{1}(A, R)\right)+l\left(\operatorname{Tor}_{2}\left(T, \operatorname{Ext}_{R}^{1}(A, R)\right)\right) \neq 0 .
$$

But on the other hand by the theorem above $\chi\left(T, \operatorname{Ext}_{R}^{1}(A, R)\right)=0$, a contradiction.

By induction on $\operatorname{dim} M$ (since, for a nonmaximal prime $p, M_{p}$ over $A_{p}$ satisfies the induction hypothesis), the proof that $A$ is C-M is completed. Finally, by Murthy's theorem on U.F.D.'s, our assertion follows.

(2) If $\operatorname{dim} \operatorname{Ext}_{R}^{1}(A, R)=0$ and $\operatorname{Ext}_{R}^{1}(A, R)^{v}$ is cyclic, then the proof of Theorem 2 shows that if $A$ is a local ring of dimension 4 possessing a module of finite injective dimension, then $A$ must be $C-M$.

NOTE. J. Koh has told me that he has also obtained a proof of indecomposibility of $H_{m}^{n-1}(A)$ in the case of a module of finite length, finite projective dimension and with depth $A=n-1$. 


\section{REFERENCES}

[AB] M. Auslander and M. Bridger, Stable module theory, Mem. Amer. Math. Soc. No. 94 (1969).

[B] H. Bass, On the ubiquity of Gorenstein rings, Math. Z. 82 (1963), 8-28.

[FFGR] R. Fossum, H. B. Foxby, P. Griffith and I. Reiten, Minimal injective resolutions with applications to dualizing modules and Govenstein modules, I.H.E.S. Publ. Math. No. 45, 1975, pp. 193-215.

[F] H. B. Foxby, On the $\mu^{i}$ in a minimal injective resolution. II, Math. Scand. 41 (1977), 19-44.

[Ha] R. Hartshorne, Residues and duality, vol. 20, Springer-Verlag, Berlin, Heideberg and New York, 1966.

[H] M. Hochster, Topics in the homological theory of modules over commutative rings, C.B.M.S. Regional Conf. Ser. Math., No. 24, Amer. Math. Soc., Providence, R. I., 1975.

[M] M. P. Murphy, A note on factorial rings, Arch. Math. 15 (1964), 418-420.

[PS] C. Peskine and L. Szpiro, Dimension projective finie et cohomologie locale, I.H.E.S. Publ. Math. No. 42, 1973, pp. 49-119.

[S] R. Y. Sharp, The construction of a module of finite projective dimension from a finitely generated module of finite injective dimension, Comment. Math. Helv. 50 (1975), 15-26.

School of Mathematics, Institute for Advanced Study, Princeton, New Jersey 08540

Current address: Department of Mathematics, University of Pennsylvania, Philadelphia, Pennsylvania 19104 